\author{
$31 \mid 2003$ \\ Les Aventures de Télémaque. Trois siècles \\ d'enseignement du français. II.
}

\title{
Pour une comparaison traductologique. Le III livre du Télémaque
}

\section{Filomena Vitale}

\section{(2) OpenEdition \\ Journals}

Édition électronique

URL : https://journals.openedition.org/dhfles/1903

DOI : $10.4000 /$ dhfles. 1903

ISSN : 2221-4038

Éditeur

Société Internationale pour l'Histoire du Français Langue Étrangère ou Seconde

Édition imprimée

Date de publication : 1 décembre 2003

Pagination : 178-186

ISSN : 0992-7654

Référence électronique

Filomena Vitale, «Pour une comparaison traductologique. Le IIIe livre du Télémaque », Documents pour I'histoire du français langue étrangère ou seconde [En ligne], 31 | 2003, mis en ligne le 01 janvier 2012, consulté le 16 juillet 2022. URL : http://journals.openedition.org/dhfles/1903 ; DOI : https://doi.org/ $10.4000 /$ dhfles. 1903

Ce document a été généré automatiquement le 16 juillet 2022.

Tous droits réservés 


\title{
Pour une comparaison traductologique. Le III livre du Télémaque
}

\author{
Filomena Vitale
}

1 Sur le sillage de la gallomanie qui sévit dans la plupart des régions européennes et italiennes au XVIII siècle, le Télémaque de Fénelon connut un énorme succès, avec des réimpressions et de très nombreuses traductions se poursuivant jusqu'à la fin du XIX ${ }^{e}$ siècle. Ces traductions n'étaient pas seulement en anglais, italien, allemand et espagnol - ce qui serait d'ailleurs assez ordinaire - mais également en portugais, polonais, suédois, roumain, russe, serbe, grec moderne et latin.

2 Puisque les catalogues (informatisés et non), ainsi que les fichiers des bibliothèques sont souvent lacunaires, il est impossible de présenter des graphiques ou des données fiables concernant la progression des éditions et traductions italiennes : c'est une opération qu'il faudra remettre à un autre moment, lorsque l'équipe italienne qui travaille sur les Télémaque aura achevé son oeuvre de repérage. Je me limiterai donc aux résultats de mes recherches dans les bibliothèques de Naples et de Potenza.

Malgré une présence non négligeable de traductions italiennes, j’ai été quelque peu déçue, en passant en revue les exemplaires conservés dans ces bibliothèques, de constater que très rarement ils s'accompagnaient de remarques linguistiques. On y rencontre en effet soit des observations pédagogiques sur les contenus édifiants de l'ouvrage, soit de brefs commentaires sur la beauté de la prose de l'auteur.

4 Les remarques stylistiques des traducteurs, souvent anonymes, ou des éditeurs, qui parfois s'exprimaient à leur place dans les textes liminaires, sont également assez rares. On est loin, bien évidemment, de véritables discours traductologiques et la qualité de ces traductions ne fait pas toujours honneur à leurs auteurs : de telles remarques semblent viser surtout à critiquer les traductions précédentes et, cela va sans dire, à justifier et à promouvoir la nouvelle. Et pourtant, il s'agit de critiques directes et ponctuelles qui, éveillant ma curiosité, ont constitué le point de départ de cette brève analyse, qui se concentre sur un seul Livre du Télémaque, le Troisième. 
5 Un tel choix a été opéré en considération de la forte présence, dans ce Livre, d'un sujet qui sera particulièrement prisé vers la fin du XVIII ${ }^{e}$ siècle : le mensonge, la

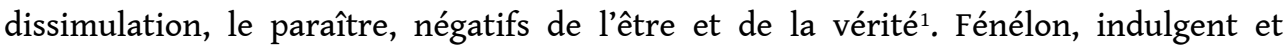
compatissant envers la finitude et l'imperfection humaines, avait représenté l'alternative à la conception culpabilisatrice de Pascal. Un siècle plus tard, ce sera la réflexion - menée sur le plan moral avant tout - de Rousseau et de Constant qui s'opposera à la rigueur kantienne.

6 Certes, ce sujet tant débattu représente une isotopie dans tout le Télémaque, mais le Troisième Livre me semblait bien se prêter à l'objectif de l'enquête: suivre l'évolution si évolution il y a - de l'art de traduire entre la deuxième moitié du XVIIIssiècle et la première du XIXe.

7 Avant d'examiner contrastivement les trois traductions prises en examen - la napolitaine, chez Gravier, de 1768, la vénitienne, chez Pasquali, de la même année, et la turinoise, chez Fontana, de 1842 - je voudrais commencer par souligner certaines affirmations contenues dans les prétentions de supériorité affichées dans les textes liminaires des deux premières.

8 Dans l'avis de l'imprimeur de l'édition de Venise (A chi legge), on trouve surtout une garantie de l'intégralité du texte traduit et du rétablissement, après tant de traductions fautives, du « vrai sens » (vero senso).

9 La même année, dans l'avis au lecteur de l'édition napolitaine (qui sera réimprimée sans variations de quelque importance jusqu'à la moitié du XIX ${ }^{e}$ siècle), on lit des critiques explicites à la traduction vénitienne :

E siccome nell'ultima Edizione del 1765 il Veneto Stampatore ne assicura d'aver procurato che fosse riveduta, e corretta sul testo, così io, volendola qui ristampare, avrei potuto riposare sulla sua fede. Ma ne sarei rimasto con mio dispiacere deluso : poiché, avendola, per camminare con maggior sicurezza, fatta confrontare coll'Originale, si è ritrovata, oltre a ogni aspettativa, piena d'errori, e in varj luoghi mancante d'alcuni periodi ed in alcun luogo mancante ancora d'intere pagine. Con questa occasione considerandosi parimenti che mal si conveniva a un'Opera cotanto ingegnosa, e cotanto vaga una traduzione, come è la Veneta, meramente verbale, $\mathrm{e}$ così abbondante di franzesismi, che ne fa perdere tutte le grazie, e fa languire quei pensieri, che maggiormente brillano nell'Originale Francese, si è per tutti questi motivi stimato di farne una nuova traduzione $[. . .]^{2}$.

Les reproches adressés à la traduction vénitienne sont donc au nombre de deux : l'excès de littéralité et la présence de gallicismes ; des défauts de forme qui affectent le contenu, les pensées, les idées.

11 Mais quel était le rapport existant, à cette époque-là, entre idées et langage ? En réalité, c'est surtout grâce à Condillac qu'une nouvelle conception du langage commençait à se frayer un chemin à côté de la conception cartésienne où il était vu comme le reflet de la réalité universelle rationnelle. Condillac avait prôné l'arbitraire du signe, puisque « les idées se lient avec les signes ${ }^{3}$ (Condillac 1946 : I, 4), et insisté sur la spécificité de chaque langue, sur la notion de relativisme des goûts et des styles des différentes langues. Voltaire aussi avait contribué à cette nouvelle vision, en diffusant le concept de "génie de la langue " ${ }^{4}$, de sorte que, à partir de la fin du XVIIIssiècle, on ne manqua pas d'examiner les problèmes de liberté et de fidélité dans les traductions à la lumière de ce concept. 


\section{de traduction, passent en Italie grâce à des personnalités telles que Francesco Algarotti} (1779) ou Melchior Cesarotti (1800).

13

, si l'on compare les deux traductions de 1768 , il est possible de faire toute une série de considérations, à partir des deux attitudes différentes adoptées par les traducteurs napolitain et vénitien, le premier « cibliste », l'autre « sourcier » (Ladmiral 1986 : 33-42). Prenons tout d'abord la traduction napolitaine : elle respecte et présente les traits caractéristiques de l'art de traduire de son siècle, le siècle des "belles infidèles ", pour reprendre l'expression utilisée par Georges Mounin dans son ouvrage consacré à la traduction aux XVII et XVIII' ${ }^{\mathrm{e}}$ siècles (Mounin 1955 : 165). Mounin soutient en effet que c'est au XVIII ${ }^{e}$ siècle que l'on a atteint la plus grande distance entre l'original et la traduction, à cause, bien sûr, du maintien des critères esthétiques classiques, l'élégance et le respect des bienséances, mais aussi de la forte tendance à l'adaptation, au " travestissement " pour paraphraser l'expression de Montesquieu (1719 : Lettre CXXVIII)

Dans l'édition napolitaine, l'on observe effectivement des traits d'ethnocentrisme culturel, si bien que, à un moment donné, les dieux se transforment en la divina provvidenza. Les procédés traductifs classiques de rationalisation, d'ennoblissement, de clarification, d'homogénéisation sont présents partout dans le texte ${ }^{6}$. Un exemple pour tous, la clarification opérée pour expliquer la puissance des Tyriens :

Voilà, sans aller chercher d'autres causes, ce qui leur donne l'empire de la mer

Senza dunque cercarne altra cagione, sono queste le arti e queste le vie, per cui tengono l'impero del mare.

Le traducteur napolitain remplace volontiers la structure paratactique de l'original par la subordination, en ayant recours aux incises et aux propositions relatives, ce qui implique forcément une introduction massive de connecteurs logiques et une forte intervention sur la ponctuation, avec suppression de points, points-virgules, deux points. Il n'y a pas abus ici de que restrictif, ni de répétitions du pronom personnel sujet, ni de l'adjectif possessif. La construction de la phrase se démarque presque toujours de la française, mais elle fausse volontiers le rythme et la linéarité de l'original. L'impression finale, en somme, est celle d'un changement de ton et de rythme, qui, en pleine harmonie avec les théories classiques, allonge, supprime, embellit, ennoblit, rationalise.

L'édition vénitienne, par contre, se révèle bien plus liée à la traduction mot à mot. Le traducteur a recours assez rarement au procédé de rationalisation, aussi bien phrastique que rythmique (et qui peut consister à modifier l'arrangement des phrases, à éliminer les redites, à adjoindre des propositions relatives ou, plus simplement, à changer la ponctuation, selon l'idée que l'on a de l'ordre du discours). Il reproduit plus fidèlement la structure paratactique de l'original, répète presque toujours le pronom personnel sujet ainsi que les adjectifs possessifs, les constructions sont souvent calquées ; son adhésion formelle à l'original va jusqu'au maintien du nom propre ${ }^{7}$ français d'Astarbé (qui garde un accent sur le $e$ final - mais un accent grave alors que dans les autres traductions c'est « Astarbe » ou " Astarbea »). Et cependant, malgré la préservation du rythme et de la fluidité de l'original, cette traduction donne l'impression d'un certain aplatissement du texte fénelonien, d'un assujettissement qui dégénère parfois en servitude. 

des choses s'étaient passées (cf. Catalano/Scotto). Tout d'abord, Fénelon était devenu une des lectures préférées des cercles libéraux du début du siècle - Coppet au premier chef - de sorte qu'il représentait, en France du moins, un point marquant de la sensibilité romantique.

côté de la réflexion sur la langue et sur la traduction, il se passe bien des choses aussi : les théories allemandes avaient imprimé une nouvelle direction à l'art de traduire en opposition avec la traduction poétisante en vogue en France à la fin du XVIII ${ }^{\mathrm{e}}$ siècle. Les concepts de fidélité profonde - pas littérale - à l'original, de nonannulation de l'étrangeté de l'original dans une pratique ethnocentrique constituent les points principaux de ce tournant décisif dans l'histoire de la traduction, et marquent son passage sur un terrain moins formel, moins extérieur (cf. Berman 1984). Tout cela a son écho en Italie à cause aussi de la polémique soulevée par le célèbre article de Mme de Staël, Sur la manière et l'utilité des traductions (1816), vulgarisé par Pietro Giordani dans la Biblioteca Italiana (1816), dont on connaît la riposte de Leopardi dans le Zibaldone (1821 : 965) .

19 Y a-t-il des traces de tout cela, dans la traduction turinoise ? En effet, il semblerait que celle-ci relève d'une conception de l'art de traduire plus affranchie de la tendance ethnocentrique d'adaptation et de l'exigence d'embellissement et que, malgré quelques fautes, le traducteur s'efforce de ne pas trahir la grâce et le style féneloniens tout en essayant de préserver la spécificité de la langue italienne. Cela ne signifie pas, certes, que l'on devra s'attendre à une fidélité à la lettre, loin de là, car il s'agit plutôt d'une fidélité à l'esprit, comme le dit Delille dans les commentaires à sa traduction de l'Énéide, une fidélité qui n'exclut pas les interventions du traducteur, lequel manifeste par exemple une certaine préférence pour la forme passive, pour le tutoiement ${ }^{9}$, pour la subordination (même si elle est modérée) et pour les sujets abstraits ou impersonnels :

Nous demeurâmes longtemps dans cette espèce de combat > Durò lungo tempo questa specie di contrasto.

Elle avait su gagner le coeur de Pygmalion par sa beauté > Era a lei riuscito con la bellezza [...] di affascinare il cuore di Pigmalione.

Pourvu que je dise toujours la vérité > Purché la verità mi sieda nel labbro.

Mais, ce qui nous frappe le plus dans cette nouvelle traduction, c'est peut-être que l'attention se concentre plus spécifiquement sur la langue d'arrivée, sur l'italien, et sur la défense de ses traits caractéristiques, de sa nature. Dans les «Cenni intorno all'autore del Telemaco ", on dénonçait en fait une connaissance approximative, de la part des traducteurs, de la langue italienne :

Molte sono le traduzioni italiane del Telemaco. Alcune di esse altro non attestano se non l'imperizia del traduttore in ambedue le favelle. Una poi, stampata in Londra e ristampata in Lombardia, pare a primo tratto assai migliore di quelle, ma esaminandola con attenzione vi si scorge che il traduttore è mille miglia lontano dal conoscere a fondo la lingua italiana, nella quale si travaglia a voler parere maestro. Ma grandemente sopra tutte si leva la traduzione anonima che ne stampò in Roma Salvioni nel 1811. Essa è certamente l'opera di qualche ingegno assai chiaro, che non volle svelarsi in un lavoro di traduzione. Non manca essa tuttavia di alcuni difetti, tra' quali, omettendo alcuni rarissimi errori nell'intelligenza del testo, spicca a nostro parere principalmente un'alterazione quasi continua nelle fattezze, se così lice dire, dello stile. Perocché il traduttore ingannandosi sull'indole della sintassi italiana, crede servire a questa, riducendo a periodi trimembri e quadrimembri i periodi incisi o bimembri dell'autore. Il $t u$, sostituito continuamente col voi dell'Autore, ci parve pure togliere qualche cosa alla grave 
amorevolezza de' discorsi di Mentore. E finalmente il numero in più luoghi potea migliorarsi (1841: VIII).

21 On retrouve ici le terme employé par Leopardi, « indole », lorsqu'il compare dans le Zibaldone les langues française et italienne - unitaire et monolithique la première, agglomérat de langues et donc accoutumée à la flexibilité la seconde - pour conclure que « la lingua italiana, benché tanto affine alla francese [...] tuttavia n'è tanto lontana e dissimile, massimamente nell'indole ; [essa] perde tutta la sua naturalezza, e la sua proprietà, o forma propria e nativa, adattandosi alla francese, che l'è pur sorella » (Leopardi $1821: 1009,1$ ).

Mais, au delà des spécificités des trois traductions, au delà des styles, des choix qui les caractérisent et les distinguent, s'il y a une chose qu'elles ont en commun, c'est un certain nombre de bévues, même grossières, dont je donnerai quelques exemples. Celles-ci sont de nature tout d'abord morphosyntaxique, déterminées parfois par la non-identification de l'antécédent d'un pronom relatif :

punissez sévèrement la fraude et même la négligence ou le faste des marchands, qui ruine le commerce en ruinant les hommes qui le font > badate a punire con severità non solamente la frode, ma la trascuragine ancora, e 'l troppo fasto di quei mercatanti, i quali, rovinando coloro, che fanno il traffico, rovinano il traffico stesso (Naples) ${ }^{10}$;

ou par un pronom relatif pris pour un interrogatif :

Insensé ! Qui ne voit pas que sa cruauté, à laquelle il se confie, le fera périr ! > Insensato! $\mathbf{E}$ chi non vede, che lo farà perire quella crudeltà medesima, in che si fida ? (Venise) ;

ou encore par le passage d'une construction parataxique à une hypotaxique, susceptible de fausser le sens de toute une phrase :

Voilà un homme qui n'a cherché qu'à se rendre heureux: il a cru y parvenir par les richesses

ecco un uomo che ha avuto unicamente in mira di rendersi felice per mezzo delle ricchezze (Turin).

Quant aux fautes de lexique, il s'agit, pour la plupart, d'imprécisions, de simples omissions, comme par exemple lorsque, dans la traduction napolitaine, la pourpre tyrienne perd son adjectif ; moins souvent on rencontre de véritables méprises, comme pour la phrase mener les hommes sans contrainte qui devient dans la traduction vénitienne pratiquement son contraire: costringere gli uomini ad ubbidire.

Il est enfin amusant et intéressant de relever les fautes imputables à une mauvaise lecture des manuscrits. Ainsi, la douleur peinte sur le visage de Télémaque devient-elle dolcezza dans la traduction turinoise (douceur pour douleur).

Et pour conclure, je voudrais m'arrêter sur deux extraits de ce Troisième Livre du Télémaque afin d'en examiner comparativement quelques éléments dans les trois traductions. J'ai choisi deux passages descriptifs - l'un du mont Liban, l'autre de la perfide Astarbé.

\section{Extrait n. 1}

Ce pays est au pied du Liban, dont le sommet fend les nues et va toucher les astres. Une glace éternelle couvre son front ; des fleuves pleins de neige tombent, comme des torrents, des pointes de rochers qui environnent sa tête. Au-dessous on voit une vaste forêt de cèdres antiques, qui paraissent aussi vieux que la terre où ils sont plantés et qui port ent leurs branches épaisses jusque vers les nues (Paris 1699, orthographe modernisée). 
Il paese è a' pié del Libano, la cui cima fende le nuvole, e va a toccare le stelle. Un ghiaccio eterno gli cuopre la fronte, ed alcuni fiumi pieni di nevi cadono, come torrenti dalle punte di que' dirupi, che gli circondano il capo. Di sotto si vede un'ampia foresta d'antichi cedri, che sembrano tanto vecchi, quanto la terra, nella quale sono piantati, e c he vanno a mettere i lor folti rami fin tra le nuvole (Venise 1768).

Questo paese è a piè del Libano, che nasconde tra le nuvole l'orgogliosa sua cima, la quale ricoperta sempre di ghiaccio dà perpetuo alimento a molti vaghi fiumi, che sgorgano come torrenti, pieni ancora di nevi tra quelle rupi. Sorge più sotto una vasta selva di folti odoriferi cedri, i quali sembrano di non cedere per l'antichità alla terra s tessa, dove sono piantati, e di voler pareggiare per altezza l'olimpo (Naples 1768).

Questo paese è situato alle falde del Libano che fende colla cima le nubi, e sembra che si spinga fino al contatto degli astri. Copre il giogo di questo monte un eterno ghiaccio ; e precipitano, a guisa di torrenti, fiumi pieni di neve dalla punta dei massi che ne circondan la vetta. Sorge più sotto una vasta foresta di cedri che sembrano non meno antichi della terra in cui sono piantati, e che sollevano i folti lor rami fino alla regione de' nembi (Turin 1842). elle savait cacher ses s entiments corrompus par un profond artifice. un profondo artificio (Venise). finissima mascherare i suoi malvagi sentimenti (Naples). profondo artifizio nascondere i corrotti suoi sentimenti (Turin). troisième. de toute langue, et, par là, de toute traduction.

Cette femme était belle comme une déesse ; elle joignait aux charmes du corps tous ceux de l'esprit ; elle était enjouée, flatteuse, insinuante. Avec tant de charmes trompeurs, elle avait, comme les Sirènes, un coeur cruel et plein de malignité ; mais

Quella donna [Astarbè] era bella, come una Dea, ed univa alle bellezze del corpo quelle altresì dello spirito : era lusinghiera, festevole, ed avea l'arte di sapersi insinuare nell'altrui grazia. Tuttavia con tanta apparenza di dolcezza aveva un cuore crudele, e pieno di malignità ; ma sape va celare i suoi sentimenti malvagi con

Era questa donna [Astarbea] non solamente bella come una Dea, ma superava ogni altra per vezzi, per lusinghe, per leggiadria. Chiudea però, come le sirene, sotto una amabile apparenza di dolcezza pensieri maligni, e pieni d'iniquità, e sapea con arte

Questa donna [Astarbe], bella come una Dea, accoppiava ai vezzi del corpo tutti quelli dello spirito. Vivace, lusinghiera, scaltra nell'insinuarsi, univa, come le sirene, a tante grazie ingannatrici un'anima crudele e maligna ; ma sapeva con

Dans le premier extrait se synthétisent et se confirment les traits caractéristiques déjà mis en évidence dans les trois traductions: la fidélité à la lettre et le respect de la parataxe pour la première - la seule qui ait gardé la métaphore du mont Liban, humanisé et doté dans l'original d'une tête et d'un front ; le penchant à l'embellissement et la préférence de la subordination - avec le conséquent emploi de nombre de relatifs et de connecteurs logiques - pour la deuxième ; l'effort de retrouver " l'équivalence dans la différence » selon la formule de Roman Jakobson (Jakobson 1963 : 78-81) pour la

Quant au deuxième extrait, il invite à réfléchir sur la dense valeur sémantique des mots, en l'occurrence du mot coeur - traduit respectivement par « cuore ", " pensieri » et, après la page romantique, par « anima »-, mot sur lequel je m'arrêterai, lui confiant ainsi la tâche de conclure cette petite enquête en tant que témoignage de l'historicité 


\section{BIBLIOGRAPHIE}

\section{Bibliographie}

ALGAROTTI, Francesco (1779), Saggio sopra la necessità di scrivere nella propria lingua, in Opere, Cremona, Manini, t. IV.

BERMAN, Antoine (1984), L'Épreuve de l'étranger. Culture et traduction dans l'Allemagne romantique, Paris, Gallimard.

------(1985), « L'analytique de la traduction et la systématique de la déformation », in Les Tours de Babel, T.E.R., Mauvezin.

CATALANO, Gabriella, SCOTTO, Fabio (2001), La nascita del concetto moderno di traduzione, Le nazioni europee fra enciclopedismo e epoca romantica, presentazione di Aurelio Principato, Roma, Armando editore.

CESAROTTI, Melchior (1945 [1800]), Saggio sulla filosofia delle lingue, Firenze, Sansoni.

CONDILLAC, Étienne Bonnot de (1946 [1746]), Essai sur l'origine des connaissances humaines, in Euvres philosophiques, v. I, G. Le Roy dir., Paris, PUF.

CONSTANT, Benjamin (1997 [1797]), Des Réactions politiques, in CEuvres complètes, t. I, Tübingen, Max Niemeyer Verlag.

FANTI, Claudia (1980), Teorie della traduzione nel Settecento italiano. Note e discussioni, Bologna, Tipografia Compositori.

FÉNELON(1768a), Le Avventure di Telemaco figliuolo di Ulisse, nuova traduzione dal francese, Napoli, a spese di Giovanni Gravier.

------(1768b), Le Avventure di Telemaco figliuolo di Ulisse, composte da Monsignor Francesco di Salignac della Mote Fenelon, Venezia, appresso Giambatista Pasquali.

------(1842), Le Avventure di Telemaco, di Fénelon, seguite dalle Avventure di Aristonoo, traduzione italiana pubblicata per cura di D.B., Torino, Stabilimento Tipografico di A. Fontana.

JAKOBSON, Roman (1963), Essais de linguistique générale, Paris, Les Éditions de Minuit.

KANT, Immanuel (1982 [1797]), Sur un prétendu droit de mentir par humanité, in CEuvres complètes, t. III, Paris, Gallimard, Bibliothèque de la Pléiade.

LADMIRAL, Jean-René (1986), « Sourciers et ciblistes », Revue d'esthétique 12, 33-42.

LEOPARDI, Giacomo (1953 [1821]), Zibaldone, in Tutte le opere, Verona, A. Mondadori.

MONTESQUIEU(1973 [1719]), Les Lettres persanes, Paris, Gallimard.

MOUNIN, Georges (1994 [1955]), Les Belles infidèles, Paris, Presses Universitaires de Lille.

PODEUR, Josiane (1999), Nomi in azione, Napoli, Liguori Editore.

STAËL, Germaine de (1975 [1816]), Sur la manière et l'utilité des traductions, in La Biblioteca italiana, a cura di Piero Oddone, Treviso, Canova, 1975.

VOLTAIRE(1926), Extraits en prose. Philosophie, Histoire, Littérature, Mélanges, Correspondance, par A. Gasté, Paris, Belin. 


\section{NOTES}

1. Je pense notamment aux Confessions de Rousseau, à sa Quatrième Rêverie mais aussi à la polémique qui a éclaté en 1797 entre Kant et Constant à propos de l'obligation morale de dire toujours la vérité.

2. Bien qu'il soit question ici de l'édition vénitienne de 1765 - dont, malheureusement, je n'ai pu trouver d'exemplaire - les critiques exprimées dans cet Avis sont si pertinentes et s'adaptent si bien à l'édition de 1768 que l'on pourrait facilement penser à une réédition.

3. Si les idées se lient aux signes, elles ne se traduisent plus rationnellement en signes, comme c'était le cas dans la vision cartésienne.

4. Voltaire (Extraits en prose 1926 : 265) donnait en fait cette définition : " On appelle génie d'une langue son aptitude à dire de la manière la plus courte et la plus harmonieuse ce que les autres langages expriment moins heureusement ».

5. On lit en effet dans cette Lettre: « Je viens de donner mon Horace au public. - Comment ! dit le géomètre, il y a deux mille ans qu'il y est. - Vous ne m'entendez point, reprit l'autre, c'est une traduction de cet ancien auteur que je viens de mettre au jour: il y a vingt ans que je m'occupe à faire des traductions. - Quoi ! Monsieur, dit le géomètre, il y a vingt ans que vous ne pensez pas ! Vous parlez pour les autres et ils pensent pour vous ? - Monsieur, dit le savant, croyez-vous que je n'aie pas rendu un grand service au public, de lui rendre la lecture des bons auteurs familière ! - Je ne dis pas tout à fait cela : j'estime autant qu'un autre les sublimes génies que vous travestissez ».

6. Ces procédés sont appelés par Antoine Berman «tendances déformantes » (1985).

7. Pour la question de la traduction des noms propres, je renvoie à Josiane Podeur (1999).

8. À Madame de Staël affirmant que le français était la langue la plus proche du latin et que les Italiens devaient s'affranchir de l'imitation des Latins et s'ouvrir à la connaissance d'autres langues, Leopardi répondait que le français était en réalité la langue romane la moins proche du latin, et que les Français connaissaient l'italien pire que toute autre langue.

9. En dépit de ce qu'on a lu dans le texte liminaire à propos de l'opportunité de garder le vouvoiement, le vous est traduit par voi aussi bien que par tu, parfois dans la bouche du même personnage s'adressant en plus au même interlocuteur (Narbale à Télémaque, par exemple).

10. Ici l'antécédent du pronom relatif est évidemment faste et non pas marchands.

\section{RÉSUMÉS}

Cette contribution entend mener une analyse comparée de trois traductions du Télémaque parues en Italie entre la deuxième moitié du XVIII ${ }^{\mathrm{e}}$ siècle et la première du XIX ${ }^{\mathrm{e}}$, car, c'est au tournant de ces deux siècles que les traductologues s'accordent à faire remonter la naissance du concept moderne de traduction en Europe. Le passage d'un art de traduire "à la française " à une nouvelle conception de la traduction, influencée par les penseurs romantiques et les idéalistes allemands, semble en effet être perceptible dans les éditions italiennes prises en examen. L'analyse du Livre Troisième permet déjà de formuler des hypothèses relatives à la façon dont ce passage a pu affecter, d'un point de vue lexical, syntaxique, prosodique et sémantique, les trois traducteurs. 
This paper presents a comparative analysis of three translations of Telemachus published in Italy between the second half of the $18^{\text {th }}$ century and the first half of the $19^{\text {th }}$ : according to translations studies, in fact, the modern conception of translation dates back to this age. Transformation from a translation « à la française » to a new conception of this art, inspired by Romantic men of letters and German Idealists, is overt in the three editions taken into consideration. After the analysis of the third book, we can formulate some hypotheses about the way in which this transformation influenced the lexical, syntactical, prosodiacal, and semantic choices in the different translations.

\section{INDEX}

Keywords : compared translation, Italy, Telemachus, Translation, XIXthsiècle, XVIIIthsiècle Mots-clés : Italie, Télémaque, Traduction, traduction comparée, XIXesiècle, XVIIIe siècle

\section{AUTEUR}

\section{FILOMENA VITALE}

Università della Basilicata 